\section{G107(P) THE INTERGROWTH-21ST NEURODEVELOPMENT PACKAGE: A NOVEL MULTI-DIMENSIONAL ASSESSMENT OF EARLY CHILD DEVELOPMENT}

${ }^{1,3} \mathrm{MC}$ Fernandes, ${ }^{2} \mathrm{~A}$ Stein, ${ }^{3} \mathrm{SH}$ Kennedy, ${ }^{3} \mathrm{~J}$ Villar. ${ }^{1}$ Department of Paediatrics, University Hospitals Southampton and University of Southampton, Southampton, UK; ${ }^{2}$ Department of Psychiatry, Warneford Hospital, University of Oxford, Oxford, UK; ${ }^{3}$ Nuffield Department of Obstetrics and Gyanecology, John Raddliffe Hospital, University of Oxford, Oxford, UK

\subsection{6/archdischild-2018-rcpch.104}

Background Globally, 250 million children under 5 years of age are at risk of not achieving their developmental potential. One of the rate-limiting steps to the scalability of child developmental assessments is their reliability on specialist professionals. The INTERGROWTH-21st Project has developed a neurodevelopment Package for rapid, holistic, and robust assessment of young children.

Objectives

i. To describe the development, characteristics and implementation of the INTERGROWTH-21st

Neurodevelopment Package

ii. To explore whether field workers from Brazil, India, Italy, Kenya and the UK can (a) administer and (b) score the INTER-NDA component of the Package comparably with healthcare professionals.

Methods The INTERGROWTH-21st Neurodevelopment Package measures (i) visual acuity and contrast sensitivity (using the Cardiff Cards) (ii) cortical auditory processing, using the auditory novelty odd-ball paradigm administered through gelfree, wireless electroencephalography (iii) cognition, language skills, motor skills, behaviour, attention and social-emotional reactivity using the 53-item INTER-NDA and (iv) sleep using actigraphy; in 22 to 26 month olds. Data are collected through a tablet-based application, the NeuroApp.

The agreement between the INTER-NDA and the BSID-III was evaluated in a sample of 81 infants from Oxford, UK. To determine whether non-specialists can implement the INTERNDA, protocol adherence and INTER-NDA domain scores were compared between field workers and healthcare professionals.

Results The administration times of the Package and the INTER-NDA are 45 and $15 \mathrm{~min}$ respectively. To date, more than 2500 children in Brazil, India, Italy, Kenya, Pakistan, South Africa, Thailand and the UK have been assessed.

Interclass correlation coefficients between the BSID-III and the INTER-NDA for cognitive, motor and behaviour domains were between 0.745 and $0.883(\mathrm{p}<0.001)$, with little to no bias in the Bland-Altman analysis.

INTER-NDA protocol adherence is $92.9 \%$ and $89.8 \%$ for healthcare professionals and field workers respectively. There are no significant differences in INTER-NDA scores between the groups.

Conclusions The INTERGROWTH-21st Neurodevelopment Package is a rapid, multi-dimensional ECD instrument. The INTER-NDA component shows good agreement with the BSID-III for the 2 year age group, despite its short administration time, and can be administered effectively by nonspecialists.

\section{G108(P) DON'T WASTE TIME! IMPROVING DOCUMENTATION FOR PLANNED PAEDIATRIC MEDICAL ADMISSIONS TO A TERTIARY CHILDREN'S HOSPITAL}

R Greeves, G Doherty, R Bothwell. General Paediatrics, Royal Belfast Hospital for Sick Children, Belfast, UK

\subsection{6/archdischild-2018-rcpch.105}

Background and aims Elective medical or specialty admissions often arrive out-of-hours when the admitting team is not available to commence care. Clearly communicating plans for investigation and management to the on-call team can prevent delays to management of these children. This quality improvement project aimed to ensure over $90 \%$ of elective medical admissions had a written plan available and treatment started within six hours by September 2017.

Methods Standard Quality Improvement methodology was used, including analysis using a driver diagram and PDSA cycles. Data was collected from the charts of electively admitted children every month and recorded whether there was a written management plan, the location of any plan, whether notes were available and clerk in completed and whether planned treatment was started within six hours. The data was analysed using a run chart. Three measurements were obtained between changes to facilitate identification of outliers. Interventions to improve compliance with admission plans included raising awareness through staff training/teaching sessions, providing written guidance to consultants and empowering the bed managers to address the whereabouts of admission plans with the responsible consultants at time of first contact.

Results Fewer elective admissions occurred over the winter period than expected as beds were occupied by emergency admissions. A large portion of elective admissions were patients admitted for sleep studies, GI procedures or investigations, metabolic investigations or planned antibiotic courses (Cystic Fibrosis). Of those studied, initially between 20\%-60\% had both a written plan available and planned treatment started within six hours. Following simple interventions 60\%$100 \%$ of patients met the standard, however, variability in the data and small numbers mean that work will need to continue to embed these changes so the results are sustained.

Conclusions Simple interventions and raising awareness improved adherence to standards for elective medical admissions, however further intervention and support is needed to make this sustainable.

\section{G109(P) ROTHERHAM RAPID ACCESS CLINIC: AN AMBULATORY CARE MODEL SERVICE EVALUATION}

H Barraclough, S Suri, D Patel, E Strawinski, J Campbell. Department of Paediatrics, Rotherham NHS Foundation Trust, Rotherham, UK

\subsection{6/archdischild-2018-rcpch.106}

Introduction Ambulatory care services such as Rapid Access Clinics (RAC) improve access to specialist secondary care and potentially reduce unnecessary referrals for acute assessment/ admission. Such clinics are endorsed by Standard 2 in Facing 